\title{
Inactivation of BLU is associated with methylation of Sp1-binding site of BLU promoter in gastric cancer
}

\author{
KUNTING XIAO $^{1 *}$, ZHUWEN YU $^{1 *}$, DONG-TAO SHI ${ }^{*}$, ZHE LEI $^{2,3}$, HONGBING CHEN $^{2,3}$, \\ JIAN CAO $^{4}$, WENYAN TIAN ${ }^{1}$, WEICHANG CHEN ${ }^{1}$ and HONG-TAO ZHANG ${ }^{2,3}$ \\ ${ }^{1}$ Department of Gastroenterology, The First Affiliated Hospital of Soochow University, \\ Medical College of Soochow University, Suzhou 215006; ${ }^{2}$ Soochow University Laboratory of Cancer Molecular Genetics, \\ Medical College of Soochow University; ${ }^{3}$ Suzhou Key Laboratory for Molecular Cancer Genetics, Suzhou 215123; \\ ${ }^{4}$ Department of Gastroenterology, The Affiliated Suzhou Municipal Hospital (Main Campus), Suzhou 215004, P.R. China
}

Received March 27, 2015; Accepted April 23, 2015

DOI: 10.3892/ijo.2015.3032

\begin{abstract}
BLU is a candidate tumor suppressor gene, which is epigenetically inactivated in many human malignancies. However, the expression and biological functions of BLU in gastric cancer has not yet been reported. In the present study, we identified a functional BLU promoter which was regulated by the transcription activator Sp1. Bisulfite sequencing and qRT-PCR assays indicated that the silence of BLU expression in gastric cancer was significantly associated with DNA hypermethylation of BLU promoter including $-39 \mathrm{CpG}$ site located in the Sp1 transcription element. The expression of BLU was notably restored in AGS and SGC7901 cells following the demethylation-treatment with 5'-Aza-2'-deoxycytidine. Moreover, the results from ChIP, EMSA and luciferase reporter gene showed that $-39 \mathrm{CpG}$ methylation could prevent $\mathrm{Sp} 1$ from binding to the promoter of BLU and decreased transcription activity of the BLU gene by $\sim 70 \%$. In addition, knockdown of BLU significantly promoted cellular proliferation and colony formation in gastric cancer cells. In conclusion, we identified a novel functional BLU promoter and proved that BLU promoter activity was regulated by $\mathrm{Sp} 1$. Furthermore, we found that hypermethylated $-39 \mathrm{CpG}$ in BLU proximal promoter directly reduced its binding with $\mathrm{Sp1}$, which may be one of the mechanisms accounting for the inactivation of BLU in gastric cancer.
\end{abstract}

Correspondence to: Dr Weichang Chen, Department of Gastroenterology, The First Affiliated Hospital of Soochow University, 188 Shizi Street, Suzhou 215006, P.R. China

E-mail: weichangchen@126.com

Dr Hong-Tao Zhang, Soochow University Laboratory of Cancer Molecular Genetics, Medical College of Soochow University, 199 Ren'ai Road, Sino-Singapore Industrial Park, Suzhou 215123, P.R. China

E-mail: htzhang@suda.edu

*Contributed equally

Key words: gastric cancer, BLU, methylation, $\mathrm{Sp} 1$

\section{Introduction}

Approximately one million people were newly diagnosed as gastric cancer patients and 738,000 new deaths were related to this malignancy in 2012 worldwide. The highest morbidity and mortality of gastric cancer are reported in countries of Eastern Asia, especially China $(1,2)$. Several high-risk etiologic factors, such as Helicobacter pylori infection, heavy salt consumption, smoking, and genetic alterations, have been proved to be associated with gastric cancer $(3,4)$. In addition, epigenetic alterations, including DNA methylation, histone modification and microRNAs, are also involved in the initiation and progression of gastric cancer $(5,6)$. Of note, promoter methylation has been considered as a common mechanism leading to the silence of tumor suppressor genes (TSGs) $(7,8)$. Since the precise mechanisms underlying the gastric tumor are still unclear, the identification of TSGs inactivated by promoter hypermethylation may provide new insights into the initiation and development of gastric cancer.

BLU was first identified by Lerman and Minna in 2000, it is also named ZMYND10 (zinc finger, MYND-type containing 10) and located at human chromosome 3p21.3 (9). The BLU protein is $\sim 50 \mathrm{kDa}$ and contains a MYND zinc finger DNA binding domain at its C-terminus, which was similar to the domains found in transcription repressors such as ETO (MTG8) and BS69 (10-12). As reported in previous studies, BLU served as a tumor suppressor in human nasopharyngeal carcinoma and ovarian carcinomas via inhibiting proliferation, inducing apoptosis and anti-angiogenic (13-16). Moreover, BLU was found frequently inactivated by the promoter hypermethylation in various human cancers, which could be restored by demethylating agent 5-Aza treatment (17-21). However, the expression and biological functions of BLU in gastric cancer remain unknown.

DNA methylation was reported to epigenetically inhibit gene expression via blocking the binding of transcription factor (TF) such as Sp1 to hypermethylated $\mathrm{CpG}$ dinucleotides within TF-binding sites (22-25). In this study, our in silico prediction has shown that two putative Sp1-binding sites are harbored in TATA-less BLU promoter, which are relative to the transcription start site of BLU. Moreover, given the fact 
that $\mathrm{Sp} 1$ is a transcription activator and Spl-binding sites are crucial for the basal transcription of genes with TATA-less promoter $(26,27)$, it is possible that the methylation of $\mathrm{CpG}$ within Sp1-binding site in the BLU promoter can lead to inactivation of BLU. Furthermore, Sp1 has been recognized to play an essential role in tumorigenesis (28). Therefore, the identification of Sp1-regulated expression of BLU may provide new clues to pathogenesis of malignancies, including gastric cancer.

Our present study supported the idea that BLU was inactivated due to its promoter hypermethylation in human cancers. However, the mechanisms by which hypermethylation results in the silencing of BLU gene have not yet been reported. In the present study, we suggest that BLU promoter hypermethylation is able to prevent $\mathrm{Sp1}$ from binding to BLU promoter in gastric cancer, and this mechanism may provide an explanation for silencing of the BLU gene.

\section{Materials and methods}

Cell culture. Six human gastric cancer cell lines (SGC7901, HGC27, BGC823, MKN45, MGC803 and AGS) and human gastric epithelial cell line GES-1 were obtained from the Cell Bank of the Chinese Academy of Sciences (Shanghai, China). SGC7901, MKN45, MGC803, and AGS were maintained in the RPMI-1640 medium (Hyclone, South Logan, UT, USA) containing $10 \%$ fetal bovine serum (FBS, Invitrogen, Carisbad, CA, USA). HGC27, BGC823 and GES-1 were cultured in the DMEM medium (Invitrogen) supplemented with $10 \% \mathrm{FBS}$. All cells were incubated at $37^{\circ} \mathrm{C}$ in a humid atmosphere of $5 \% \mathrm{CO}_{2}$.

Tissues samples. Fifty-two paired gastric cancer tissues and adjacent non-cancerous gastric tissues were obtained after informed consents from patients at the First Affiliated Hospital of Soochow University. Tissue samples were collected, immediately snap-frozen in liquid nitrogen and stored in $-80^{\circ} \mathrm{C}$ until analysis. None of the patients had received chemotherapy or radiotherapy prior to surgery. This research was approved by the Academic Advisory Board of Soochow University.

Demethylating agent 5-Aza treatment. 5-Aza was purchased from Sigma-Aldrich Corp. (St. Louis, MO, USA) and dissolved in sterile double distilled water. Cells were plated at a density of $1 \times 10^{5}$ cells per well in the 6 -well plates and incubated overnight. On days 1, 3 and 5, the medium was changed to serum-free RPMI-1640 containing $10 \mu \mathrm{M}$ 5-Aza. On days 2 and 4, the medium was changed to 5-Aza free RPMI-1640 with $3 \%$ FBS. The cells were harvested for further study on day 5 .

Quantitative real-time reverse transcription PCR ( $q R T-P C R)$. Total RNA was extracted from cells using RNAiso Plus (Takara Biotech Co., Ltd., Dalian, China) according to the manufacturer's protocol. Total mRNA $(1 \mu \mathrm{g})$ was reversetranscribed by the M-MLV reverse transcriptase (Invitrogen). Subsequently, aliquots of cDNA were subjected to PCR by using FastStart Universal SYBR Green Master (ROX) (Roche, Basel, Switzerland) on a LightCycler ${ }^{\circledR} 96$ Real-Time PCR System (Roche). Primers for mRNA detection of BLU, Sp1 and $\beta$-actin (internal control) were as follows: for BLU, 5'-AAC CAGCAGCATGAGAACCT-3' (forward) and 5'-AGTTTGCG GTGGCAATAGTC-3' (reverse); for Sp1, 5'-CCTCCAGACC ATTAACCTCAG-3' (forward) and 5'-TCCACCTGCTGTGT CATCAT-3' (reverse); for $\beta$-actin, 5'-CACAGAGCCTCGCCTT TGCC-3' (forward) and 5'-CACATGCCGGAGCCGTTGTC-3' (reverse). The amplification conditions were as follows: $95^{\circ} \mathrm{C}$ for $10 \mathrm{~min}$, followed by 40 cycles of $95^{\circ} \mathrm{C}$ for $10 \mathrm{sec}$ and $60^{\circ} \mathrm{C}$ for $30 \mathrm{sec}$. Relative fold-change in gene expression was determined using the $\Delta \Delta \mathrm{Ct}$ method normalized to $\beta$-actin.

Bisulfite sequencing. Genomic DNA was extracted from cells using DNeasy Blood and Tissue kit (Qiagen, Hilden, Germany) and then bisulfited using EpiTect Bisulfite kit (Qiagen) according to the manufacturer's instructions. The sodium bisulfite-modified genomic DNA was subjected to PCR amplification with primers which specially designed to analysis the methylation status of $22 \mathrm{CpG}$ sites within a 244-bp region of the BLU promoter (-181 to +63$)$. The primers were: 5'-ATAAG GATTTGGAGTTTAGGAGAG-3' (forward) and 5'-CCAAAA TCTAAAACAAAACAATTAC-3' (reverse). PCR products were purified by Gel Extraction kit (Omega, USA) and cloned into pMD19-T vector (Takara) for DNA sequencing. At least ten clones from each sample were randomly selected and then sequenced.

Western blot assay. Cells were lysed in RIPA buffer (Cell Signaling Technology) with the protease inhibitor (SigmaAldrich Corp.). Equal amounts of total protein were separated on SDS-PAGE and transferred to the nitrocellulose membranes (Millipore, Billerica, MA, USA). The membranes were blocked with $3 \%$ BSA-TBST buffer for $1 \mathrm{~h}$ at room temperature and then incubated with appropriate primary antibodies overnight at $4^{\circ} \mathrm{C}$. Next, the membranes were washed with TBST buffer three times and incubated with the corresponding HRP-conjugated secondary antibodies for $2 \mathrm{~h}$ at room temperature. The specific protein band detection was performed using a commercial ECL kit (Pierce, Rockford, IL, USA). $\beta$-actin was used as internal control. The following antibodies were used for western blot analysis: rabbit anti-BLU polyclonal antibody (ab96700, 1:2,000, Abcam, Cambridge, UK); rabbit anti-Sp1 polyclonal antibody (\#9389S, 1:1,000, Cell Signaling Technology, Danvers, MA, USA); rabbit anti- $\beta$-actin polyclonal antibody (sc-130656,1:1,000, Santa Cruz Biotechnology, Santa Cruz, CA, USA).

Chromatin immunoprecipitation (ChIP) assay. The ChIP assay was performed using EZ-ChIP ${ }^{\mathrm{TM}}$ Chromatin Immunoprecipitation kit (Millipore). Immunoprecipitation was performed by the addition of anti-Sp1 antibody, and rabbit IgG antibody was used as a negative control. A fragment of the BLU promoter $(-80 /+108)$, which contains putative Spl-binding sites (-43/-35) was amplified by semi-quantitative PCR with primers as follows: 5'-TAGAGACCCGCCCGGAT TTA-3' (forward) and 5'-GGGTGGGGATGCTGTCACAT-3' (reverse). PCR products were analyzed by electrophoresis on a $2 \%$ agarose gel and stained with ethidium bromide.

Electrophoresis mobility shift assay (EMSA). Nuclear extracts were isolated from cells using Nuclear Protein Extraction kit 
Table I. siRNA sequences used for silencing of Sp1 and BLU.

\begin{tabular}{|c|c|}
\hline Tame & Sequences $\left(5^{\prime}-3^{\prime}\right)$ \\
\hline 1 & $\begin{array}{l}\text { Sense: } \\
\text { 5'-GAUCACUCCAUGGAUGAAATT-3' } \\
\text { Antisense: 5'-UUUCAUCCAUGGAGUGAUCTT-3' }\end{array}$ \\
\hline$-\mathrm{Sp} 1$ & $\begin{array}{l}\text { Sense: } \\
\text { 5'-GCAACAUGGGAAUUAUGAATT-3' } \\
\text { Antisense: 5'-UUCAUAAUUCCCAUGUUGCTT-3' }\end{array}$ \\
\hline RIJU & $\begin{array}{l}\text { Sense: } \\
\text { Antisense: 5'-CCUCCAUCAUACAAGCCUCUGCACUGCTT-3' }\end{array}$ \\
\hline i-BLU-2 & $\begin{array}{lr}\text { Sense: } & \text { 5'-GCAGUGCAGAGGCUUCUAATT-3' } \\
\text { Antisense: 5'-UUAGAAGCCUCUGCACUGCTT-3' }\end{array}$ \\
\hline -BLU-3 & $\begin{array}{l}\text { Sense: } \quad \text { 5'-CCCUCUCAGUACUACGCUATT-3' } \\
\text { Antisense: 5'-UAGCGUAGUACUGAGAGGGTT-3' }\end{array}$ \\
\hline
\end{tabular}

(Solarbio, Beijing, China). The EMSA assay was performed by LightShift Chmiluminescent EMSA kit (Pierce) following the manufacturer's instructions. The 5' biotin-labeled and double-stranded oligo probes containing putative Sp1 binding sites (-43/-35) were chemically synthesized by Invitrogen. The sequences of probes were as follows: -39C (unmethylated) 5'-CCTCCAGGGGCGGGGCCCAGTTG-3' and -39 5mC (methylated) 5'-CCTCCAGGGG5mCGGGGCCCAGTTG-3'; unlabeled probes 5'-CCTCCAGGGGCGGGGCCCAGTTG-3' were synthesized as cold competitor. Electrophoresis mobility gel shift assay was performed as described previously (29).

Plasmid construction, transfection and luciferase report assays. The pGL3-basic dual luciferase vector (Promega, Madison, WI, USA) was used to produce a series of constructs containing various truncated regions of BLU promoter. Two fragments of interest BLU promoter regions containing the unmethylated and methylated $-39 \mathrm{CpG}$ (5'-CCTCCAGGGG CGGGGCCCAG-3' and 5'-CCTCCAGGGG5mCGGGGCC CAG-3', respectively) were directly chemically synthesized and massively inserted into the luciferase pGL3-basic vector, and then purified by using Universal DNA purification kit (Tiangen, Beijing, China). To assess promoter activity, cells were transiently co-transfected with BLU promoter constructs and Renilla phRL-TK plasmid (Promega) using Lipofectamine 2000 (Invitrogen). Twenty-four hours later, cells were harvested, and luciferase activities were measured by the Dual-Luciferase Reporter assay kit (Promega) on a TD20/20 Luminometer (Turner Designs, Sunnyvale, CA, USA). Results are expressed as relative luciferase activities, which are normalized to Renilla luciferase activities.

RNA interference experiments. Small interfering RNA (siRNA) sequences (Table I), which target Sp1 and BLU, were chemically synthesized (GenePharma, Shanghai, China). Scrambled siRNA was used as a negative control (NC). Cells were transiently transfected with 100 pmol of siRNA sequences using Lipofectamine 2000 (Invitrogen). After 72-h transfection, the cells were harvested for further experiments.
Cell proliferation. Cell viability was measured by a cell Counting Kit-8 (CCK-8) (Yeasen, Shanghai, China). After being transfected with or without siRNA as described above, $100 \mu \mathrm{l}$ SGC7901 and AGS cell suspensions $\left(2 \times 10^{4}\right.$ cells $\left./ \mathrm{ml}\right)$ were plated into each well of 96-well plates. Then $10 \mu \mathrm{l} \mathrm{CCK-8}$ solution was added to each well and incubated for $2 \mathrm{~h}$. The absorbance was measured at $450 \mathrm{~nm}$ on a microplate reader (MRX, Dynex Technologies, West Sussex, UK).

Colony formation assay. Two thousand cells were plated in $60-\mathrm{mm}$ culture dishes and incubated at $37^{\circ} \mathrm{C}$ for 2 weeks; visible cell colonies were fixed with methanol for $15 \mathrm{~min}$, then stained with Giemsa for $1 \mathrm{~h}$, washed with water and colonies containing $\geq 50$ cells were counted under a microscope.

Statistical analysis. Statistical analysis was performed using the SPSS 19.0 software (SPSS Inc, Chicago, IL, USA). All quantitative data were obtained from at least three independent experiments and represented as means \pm SD or SEM. Difference between the means of two groups was compared using Student's t-tests. Comparisons between clinicopathologic characteristics and expression levels of mRNA in gastric cancer samples were performed with non-parametric tests (Mann-Whitney U test for 2 groups, Kruskall-Wallis test for $\geq 3$ groups). $\mathrm{P}<0.05$ was considered to be statistically significant.

\section{Results}

BLU expression is reduced in gastric cancer cells and tissues. To verify whether inactivation of BLU contributes to gastric carcinogenesis, we first detected BLU mRNA expression in human gastric cancer cells and GES-1 cells. As shown in Fig. 1A, BLU mRNA was significantly lower in MGC803, SGC7901, BGC823, MKN45, AGS and HGC27 cells than that in GES-1 cells $(\mathrm{P}<0.05, \mathrm{P}<0.01, \mathrm{P}<0.001, \mathrm{P}<0.001, \mathrm{P}<0.001$ and $\mathrm{P}<0.001$, respectively). Consistently, BLU protein levels were also downregulated in gastric cancer cells (Fig. 1B). In addition, BLU mRNA expression was notably reduced in 69\% (36/52) gastric cancer tissues when compared with their paired adjacent non-cancerous tissues $(\mathrm{P}<0.01$, Fig. $1 \mathrm{C}$ and $\mathrm{D})$. No significant difference in BLU mRNA level was observed between gastric cancer tissues when classified by various clinicopathologic characteristics (Table II).

Identification and characterization of a functional $C p G$-rich $B L U$ promoter. To define the BLU promoter, a 2,100-bp sequence, including BLU proximal promoter $(-2,000-+1))$ and 5'-UTR (+1-+100) regions (RefSeq: NC_000003.12 and NM_015896), was available for analysis. A 251-bp region spanning positions -290 to -40 relative to the transcription start site was predicted to be the putative promoter of the BLU gene using the Promoter Scan program (Fig. 2A). This region contains a GC box, but lacks a TATA box. A potential CpG island spanning positions -574 to +90 was identified by Methyl Primer Express ${ }^{\circledR}$ software v1.0 (Applied Biosystems) (Fig. 2A).

To evaluate the effects of this sequence on transcription, we generated the luciferase report constructs containing various lengths of the BLU proximal promoter and 5'-UTR 
A

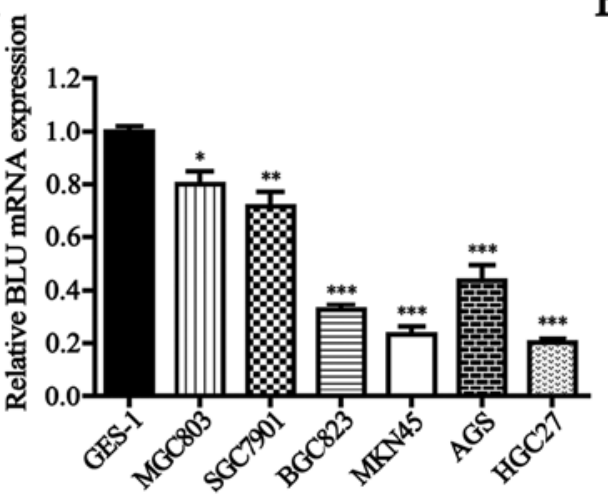

D
B

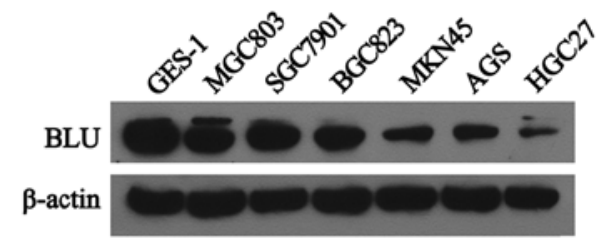

$\mathrm{C}$

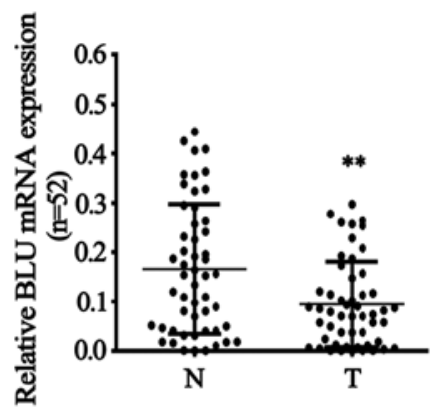

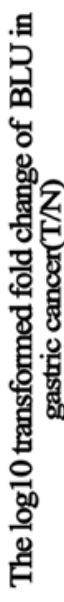

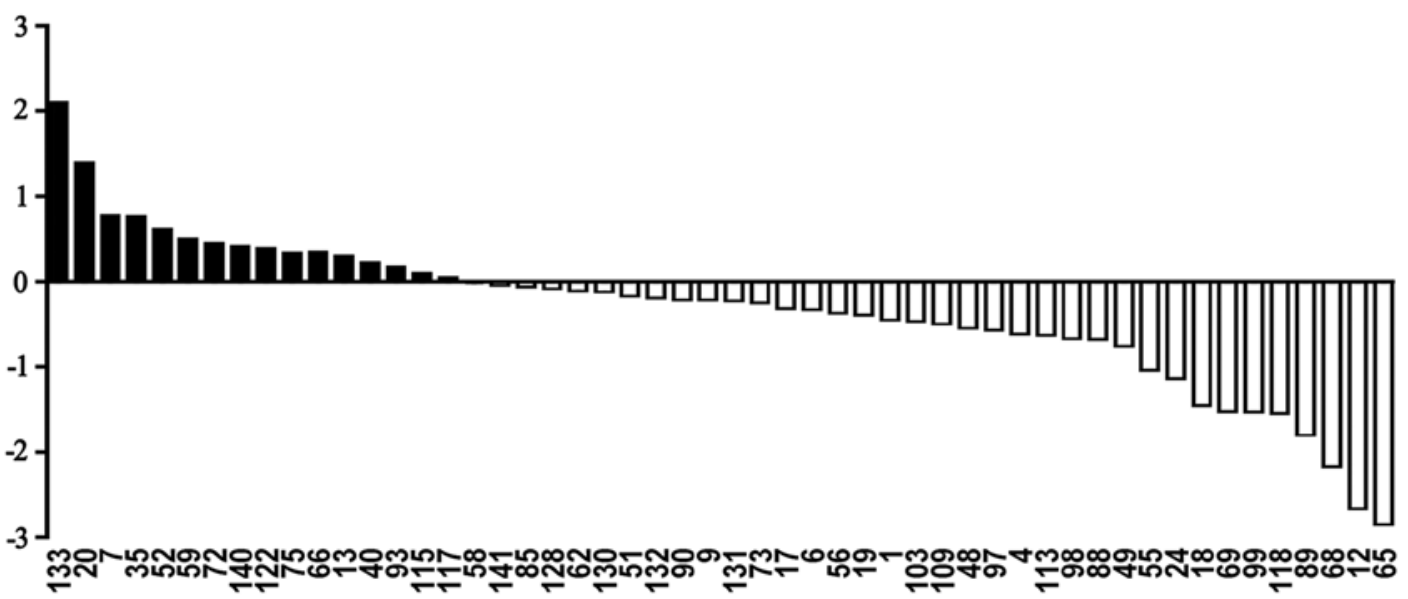

Figure 1. BLU expression is reduced in human gastric cancer cells and tissues. (A and B) The mRNA and protein levels of BLU expressed in gastric epithelial cell line GES-1 and gastric cancer cell lines MGC803, SGC7901, BGC823, MKN45, AGS and HGC27. (C and D) Expression of BLU mRNA in 52 gastric cancer tissues $(\mathrm{T})$ and paired non-cancerous gastric mucosa $(\mathrm{N})$, Y-axis represents the $\log _{10}$ transfold change of T/N mRNA expression ratio of BLU. The number of each sample is indicated below the $\mathrm{x}$-axis. ${ }^{*} \mathrm{P}<0.05 ;{ }^{* *} \mathrm{P}<0.01 ;{ }^{* * *} \mathrm{P}<0.001$.

A

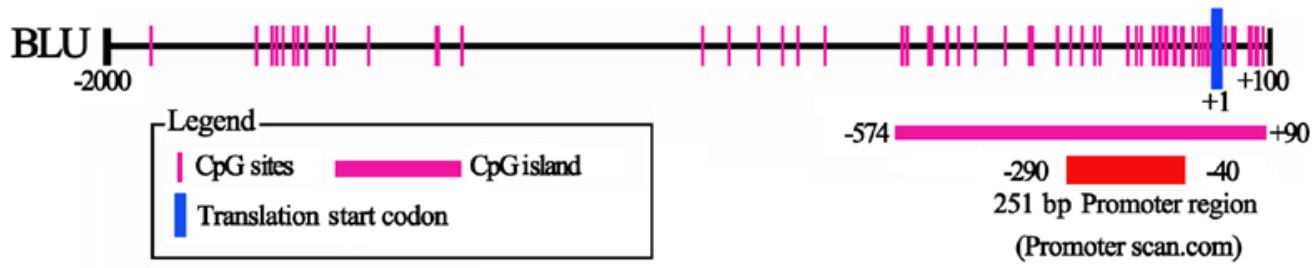

B
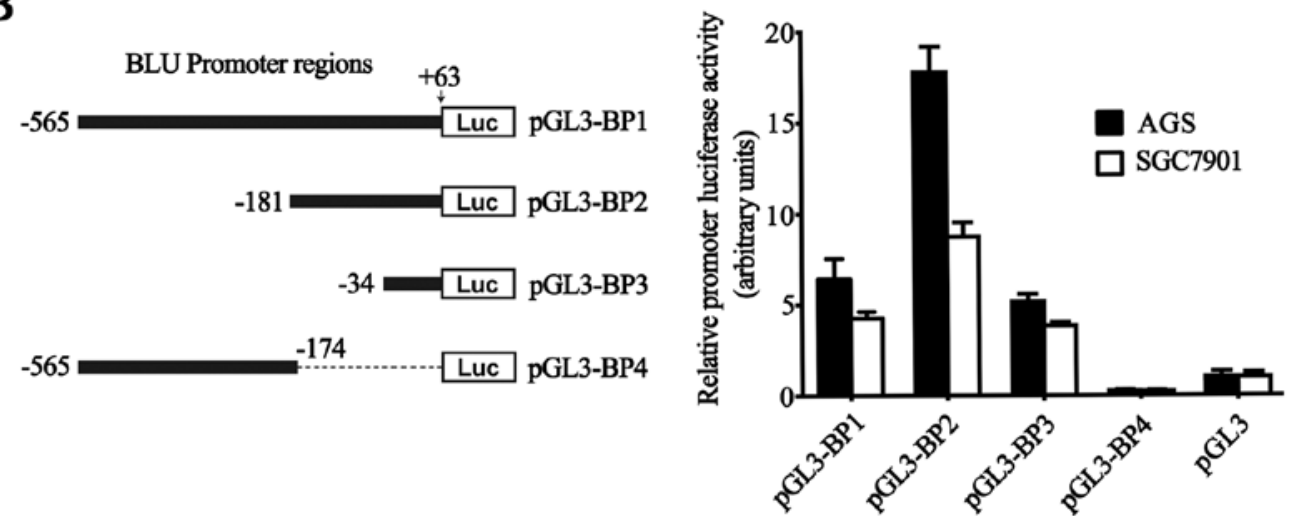

Figure 2. Identification of a putative promoter of the BLU gene. (A) Schematic of the putative BLU promoter and CpG islands. Each vertical bar represents one $\mathrm{CpG}$ site. The pink and red rectangle boxes represent a $\mathrm{CpG}$ island and a putative promoter region, respectively. The transcription start site is defined as position +1 , and the rest of the sequence is numbered relative to the transcription start site. (B) Relative luciferase activities of plasmids (pGL3-BP1, -BP2, -BP3 and -BP4) containing various lengths of the BLU promoter in AGS and SGC7901 cells. The relative luciferase activities of these constructs are shown as bar in the histograms (right panel) and each construct is shown (left panel). 
Table II. Correlation between clinicopathological features and expression levels of BLU mRNA in gastric cancer.

\begin{tabular}{|c|c|c|}
\hline Characteristic & Case $(\mathrm{n}=52)$ & BLU mRNA \\
\hline \multicolumn{3}{|l|}{ Gender } \\
\hline Male & 35 & $0.095 \pm 0.015$ \\
\hline Female & 17 & $0.091 \pm 0.018$ \\
\hline Z score ${ }^{a}$ & & -0.146 \\
\hline P-value & & 0.884 \\
\hline \multicolumn{3}{|l|}{ Age (year) } \\
\hline$\geq 60$ & 28 & $0.084 \pm 0.015$ \\
\hline$<60$ & 24 & $0.105 \pm 0.018$ \\
\hline $\mathrm{Z}$ score $^{\mathrm{a}}$ & & -0.661 \\
\hline P-value & & 0.509 \\
\hline \multicolumn{3}{|c|}{ Lymphatic invasion } \\
\hline Absent & 34 & $0.099 \pm 0.015$ \\
\hline Present & 18 & $0.084 \pm 0.020$ \\
\hline Z score ${ }^{a}$ & & -0.587 \\
\hline P-value & & 0.557 \\
\hline \multicolumn{3}{|c|}{ WHO classification } \\
\hline $\mathrm{W} / \mathrm{D}$ & 20 & $0.092 \pm 0.019$ \\
\hline $\mathrm{M} / \mathrm{D}$ & 17 & $0.094 \pm 0.020$ \\
\hline $\mathrm{P} / \mathrm{D}$ & 15 & $0.081 \pm 0.020$ \\
\hline $\mathrm{H}$ score $^{\mathrm{b}}$ & & 0.077 \\
\hline P-value & & 0.962 \\
\hline \multicolumn{3}{|l|}{ TNM stage } \\
\hline I & 19 & $0.090 \pm 0.019$ \\
\hline II & 14 & $0.093 \pm 0.025$ \\
\hline III & 11 & $0.076 \pm 0.029$ \\
\hline IV & 8 & $0.075 \pm 0.019$ \\
\hline $\mathrm{H}$ score $^{\mathrm{b}}$ & & 0.341 \\
\hline P-value & & 0.952 \\
\hline
\end{tabular}

Data are presented as means \pm SE. ${ }^{\mathrm{a} M a n n}$-Whitney $\mathrm{U}$ test for 2 groups. ${ }^{\mathrm{b}}$ Kruskal-Wallis test for $\geq 3$ groups. W/D, well-differentiated adenocarcinoma; M/D, moderately differentiated adenocarcinoma; $\mathrm{P} / \mathrm{D}$, poorly differentiated adenocarcinoma.

region. As presented in Fig. 2B, the fragment $(-181$ to +63$)$ had the highest activity, while the second largest fragment $(-565$ to -174$)$ had less activity than the largest and smallest fragments ( -565 to $+63 ;-34$ to +63 , respectively) (Fig. $2 \mathrm{~B})$, suggesting that an active cis-element was putatively at position -181 to +63 and a repressive element resides was located between positions -565 to -174 in the BLU promoter. Taken together, the results indicated that the indentified BLU promoter is indeed functional.

In silico prediction of functional $C p G$ sites in BLU promoter. Using Methyl Primer Express software (Applied Biosystems), we identified that the 244-bp BLU promoter region (-181 to +63 ) contains $22 \mathrm{CpG}$ sites (Fig. 3A). Using the transcrip- tion factor search program TFSEARCH (http://mbs.cbrc.jp/ research/db/TFSEARCH.html), two putative transcription factor Sp1-binding sites were predicted at position -43/-35 and $-89 /-81$ relative to the transcription start site, each of them contains one $\mathrm{CpG}$ site ( $-39 \mathrm{CpG}$ and $-85 \mathrm{CpG}$, respectively). Binding sites for several other potential transcription factor, such as IK-2 (one site), CdxA (two sites), GATA-1 (one site), E2F (one site), MZF1 (one site) were also noted (Fig. 3A). Since the BLU promoter lacks TATA box and the binding sites of transcription factor Sp1 was reported to be crucial for the basal transcription of TATA-less promoters, we then investigated the putative functions of $-39 \mathrm{CpG}$ and $-85 \mathrm{CpG}$ in the regulation of BLU expression.

The BLU promoter is hypermethylated in gastric cancer. We performed bisulfite sequencing (BSP) analysis to investigate whether promoter $\mathrm{CpG}$ methylation is associated with the loss of BLU expression. As shown in Fig. 3B, the hypermethylation of BLU was detected in AGS, MKN45, HGC27, BGC823 and SGC7901 cells, while BLU was hypomethylated in GES-1. Importantly, we observed that the methylation level of $-39 \mathrm{CpG}$ is significantly higher in the five gastric cancer cell lines with relatively low BLU mRNA expression (Fig. 3B-D). In addition, we also found a negative correlation between $-39 \mathrm{CpG}$ methylation level and BLU expression in tumor samples (Fig. 3E and F). Furthermore, we treated AGS and SGC7901 cells with demethylating agent 5-Aza, and found that BLU mRNA and protein levels were restored in AGS and SGC7901 cells (Fig. 3G). These results implied that hypermethylation of BLU promoter may play an important role in transcription silencing of the BLU gene in human gastric cancer.

Spl specifically binds to the BLU promoter and hypermethylation of $-39 \mathrm{Cp} G$ decreases its binding ability. It is well known that $\mathrm{Sp} 1$ is one of the first identified eukaryotic transcription factors (30). Hypermethylation of $\mathrm{CpG}$ dinucleotides within Sp1-binding sites could block Spl binding to promoter $(22,23)$. Being in silico predicted to be within Spl-binding sites in BLU promoter, the $-39 \mathrm{CpG}$ was hypermethylated in 5 gastric cancer cell lines with low BLU mRNA expression. Thus, we suggest the possibility that hypermethylated $-39 \mathrm{CpG}$ may affect the recruitment of Spl to BLU promoter. To test this, we performed the following experiments.

First, western blot analysis showed that Sp1 protein expression in SGC7901 cells was nearly equaled with that in AGS cells (Fig. 4A). Subsequently, we performed ChIP assay in AGS and SGC7901 cells. As shown in Fig. 4B, with the DNA samples immunoprecipitated by anti-Sp1 as templates, a 188 bp BLU promoter sequence $(-80$ to $+108 \mathrm{bp})$ containing -39 CpG was amplified in SGC7901 cells, but not in AGS cells. Considering that the methylation level of $-39 \mathrm{CpG}$ in AGS cells was higher than SGC7901 cells (Fig. 3B and C), the results suggested that hypermethylation of $-39 \mathrm{CpG}$ significantly inhibited the interaction between Sp1 and BLU promoter.

Next, EMSA assay showed that although both the probe containing unmethylated $-39 \mathrm{CpG}$ and the probe containing methylated -39 CpG could form DNA-protein complexes with nuclear extracts of SGC7901 cells, binding ability of 
A

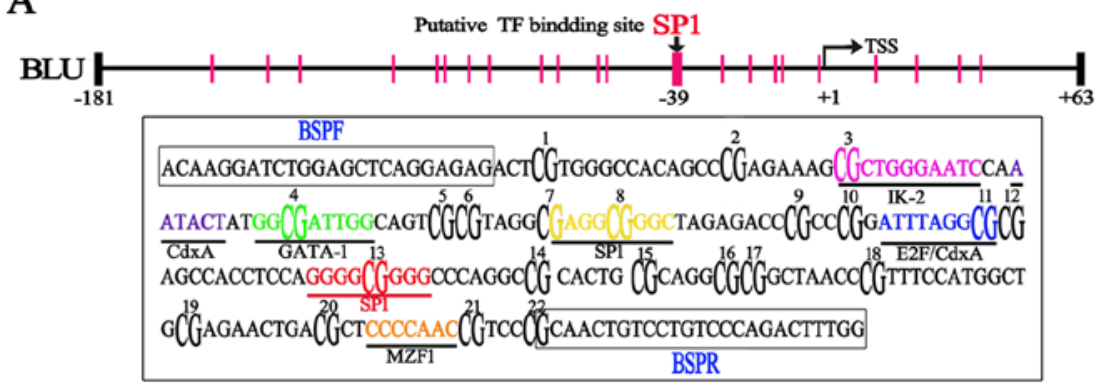

B

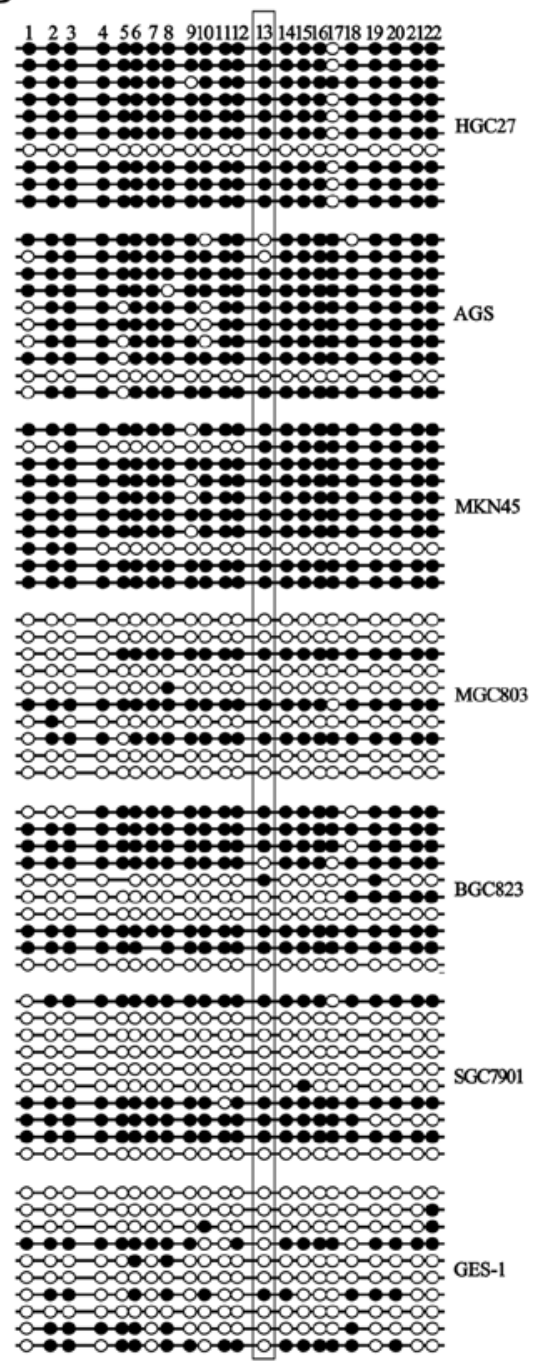

C

Sequence before bisulfite modification

CGCGAGTTAT TT TTAGGGGTGGGG TTTAGGTCC

Presence of methylation

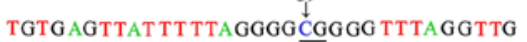

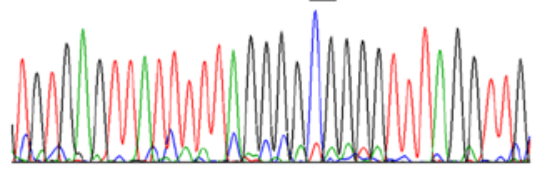

Absence of methylation

CGCGAGTTAT TT TTAGGGG TGGGG TTTAGGTCG

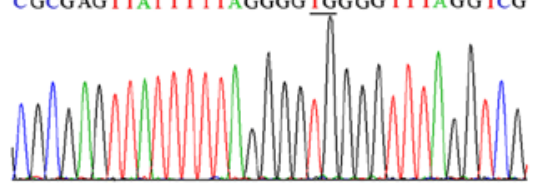

D

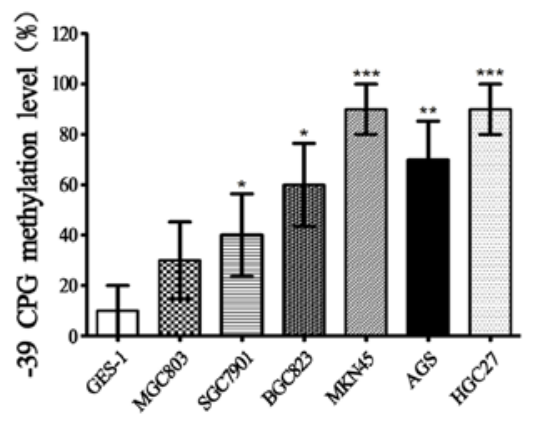

E

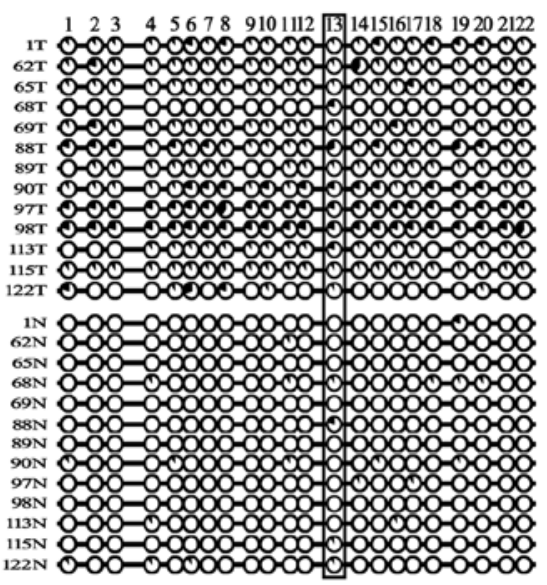

F

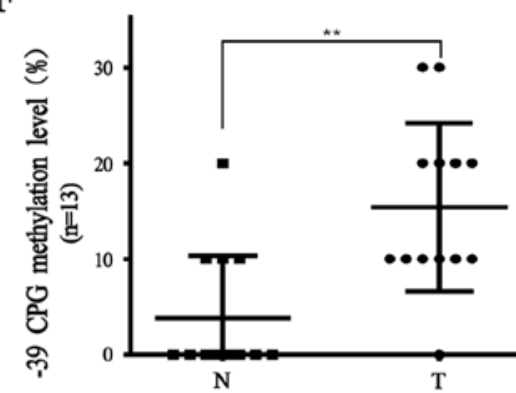

G
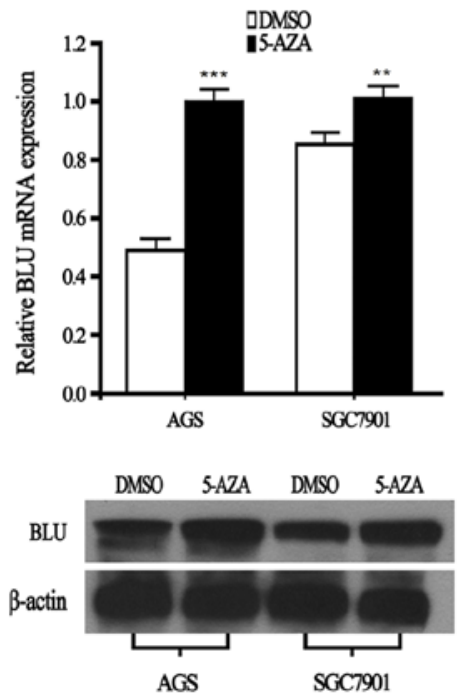

Figure 3. Characterization of BLU promoter, and methylation level of BLU promoter in gastric cancer. (A) Locations of 22 CpG sites analyzed in the BLU promoter, and each vertical bar represents one CpG site. Sequence of the BLU promoter (-181 to +63) is boxed below. The putative binding sites for IK-2, CdxA, GATA-1, E2F, Sp1, and MZF1 are labeled and shown in different colors, CpG sites are bold and numbered according to the order used in bisulfite genomic sequencing, BSP primer sequences (BSPF and BSPR) are boxed. (B) BSP analysis for methylation of cytosine residues in $22 \mathrm{CpG}$ sites at the BLU promoter in gastric cancer cell lines and gastric epithelial cell line GES-1. Methylation analysis was performed in 10 clones. Open or filled circles represent unmethylated or methylated cytosine residues, respectively. (C) Representative sequences showing the presence or absence of methylation at $-39 \mathrm{CpG}$ site. Unmethylated nucleotide $\mathrm{C}$ is converted to $\mathrm{T}$ after bisulfite treatment, but methylated $\mathrm{C}$ remains unchanged. (D) Summary of $-39 \mathrm{CpG}$ methylation level in all the cell lines. At $-39 \mathrm{CpG}$ site, the methylation level was calculated as the ratio of methylated clones over the total number of clones sequenced per cell line. (E and $\mathrm{F}$ ) Methylation status of BLU promoter in 13 randomly selected gastric tissues $(\mathrm{N})$ and their paired non-cancerous gastric tissues $(\mathrm{T})$, methylation analysis was performed in 10 clones, the percentage of methylation is indicated as pie charts. (G) The expression of BLU mRNA and protein in AGS and SGC7901 cells treated with or without 5-Aza $(10 \mu \mathrm{M}) .{ }^{*} \mathrm{P}<0.05 ;{ }^{* * *} \mathrm{P}<0.01 ;{ }^{* * * *} \mathrm{P}<0.001$.

unmethylated probe is stronger than the methylated probe (Fig. 4C). This indicated that methylation of $-39 \mathrm{CpG}$ within Sp1 binding-sites may reduce the complex formation.
Furthermore, we constructed the luciferase reporter vectors containing the corresponding unmethylated and methylated fragments (Sp1-binding sites) and then transiently transfected 
A

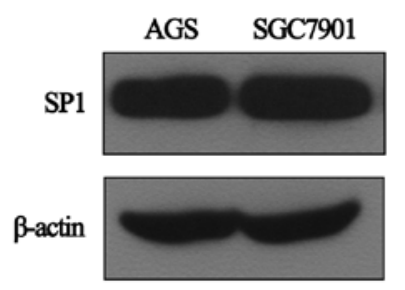

C

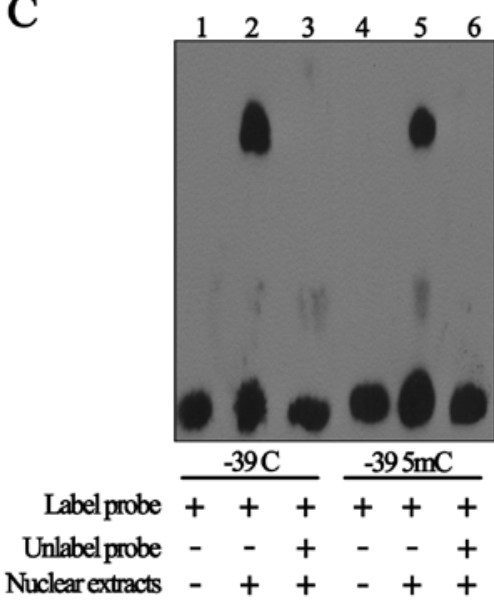

B

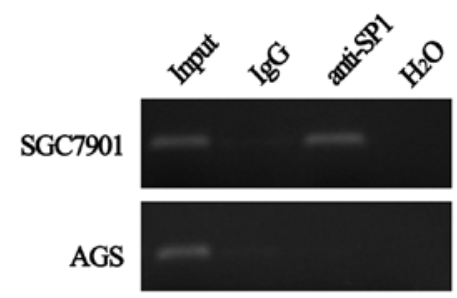

D

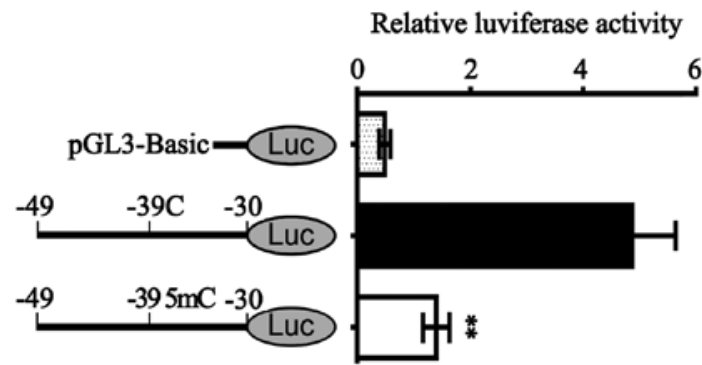

-39 C: 5'-CCTCCAGGGGCGGGGCCCAG-3'

$-395 \mathrm{mC}:$ ' '-CCTCCAGGGG5mCGGGGCCCAG-3'

Figure 4. Sp1 specifically binds to the BLU promoter, and -39 CpG was crucial for the binding activity. (A) Western blot analysis for Sp1 protein level in AGS and SGC7901 cells. (B) ChIP assay was performed using anti-Sp1 antibody to identify Sp1-binding sites in the BLU promoter in AGS and SGC7901 cells. Presence of BLU promoter sequence (positions -80 to +108 ) containing $-39 \mathrm{CpG}$ prior to immunoprecipitation was confirmed by PCR ( $1 \%$ input). IgG antibody was used as a negative control. (C) EMSA assay was completed with biotin-labeled oligonucleotide probes and cell nuclear extracts from SGC7901 cells. The biotin-labeled probes were incubated in the absence (lanes 1 and 4) or presence of nuclear extracts (lanes 2 and 5). For the competition experiment, 200-fold excess unlabeled -39 C probes (lane 3) and -39 $5 \mathrm{mC}$ probes (lane 6) were used to determine the binding sequence specificity. (D) Effect of methylated -39 CpG on transcriptional activity of BLU promoter. The constructs carrying the unmethylated or methylated $-39 \mathrm{CpG}$ were transiently transfected into SGC7901 cells and subjected to luciferase reporter assay. ${ }^{* *} \mathrm{P}<0.01$.

them into SGC7901 cells. As shown in Fig. 4D, methylated -39 CpG significantly diminished the luciferase activity by $\sim 70 \%$. Taken together, our results demonstrated that Sp1 could specifically bind with the BLU promoter and hypermethylation of $-39 \mathrm{CpG}$ significantly reduced the recruitment of Sp1 to BLU promoter.

Spl activates the BLU promoter. To explore whether Sp1 could regulate transcriptional activity of the BLU promoter, we performed the luciferase report assay in SGC7901 cells. As a result, the constructs containing the Sp1-binding site (BP5: $-59 /+63$ ) showed significant higher activity than the constructs with deletion of Sp1-binding site (BP3: -34/+63) and the mutant constructs (BP6: -59/+63) (Fig. 5A). Furthermore, siRNA-mediated knockdown of endogenous Sp1 effectively decreased the mRNA and protein expression level of BLU (Fig. 5B and C). After co-transfection of the BLU promoter constructs (BP2: $-181 /+63$ and BP5: -59/+63) containing the Sp1-binding sites with si-Sp1, knockdown of Sp1 decreased the luciferase activities of these constructs when compared with the negative control (NC) (Fig. 5D). By contrast, downregulation of Sp1 presented no significant effects on the luciferase activity of the construct
(BP6: $-34 /+63$ ) lacking the Sp1-binding sites. Our results demonstrated that the sequence of the BLU promoter (positions -43 to -35 ) is a functional Sp1-binding site and Sp1 could activate the BLU promoter.

Knockdown of BLU promotes the growth of gastric cancer cells. BLU was frequently downregulated in gastric cancer cells and primary tumors, suggesting that BLU is likely a tumor suppressor in gastric cancer. To investigate the affects of BLU on the growth of gastric cancer cells, we performed CCK-8 and colony formation assays in SGC7901 cells transfected with BLU siRNAs or negative control. As expected (Fig. 6A), BLU was significantly silenced in SGC7901 cells transfected with si-BLU-2 and si-BLU-3. Subsequently, CCK-8 and colony formation experiment showed that BLU knockdown promoted cell viability and clonogenicity (Fig. 6B and C), suggesting that BLU acts as a tumor suppressor via inhibiting cell growth in gastric carcinoma.

\section{Discussion}

Gastric carcinoma is the fifth most common cancer and second leading causes of cancer related deaths worldwide (1). During 
A
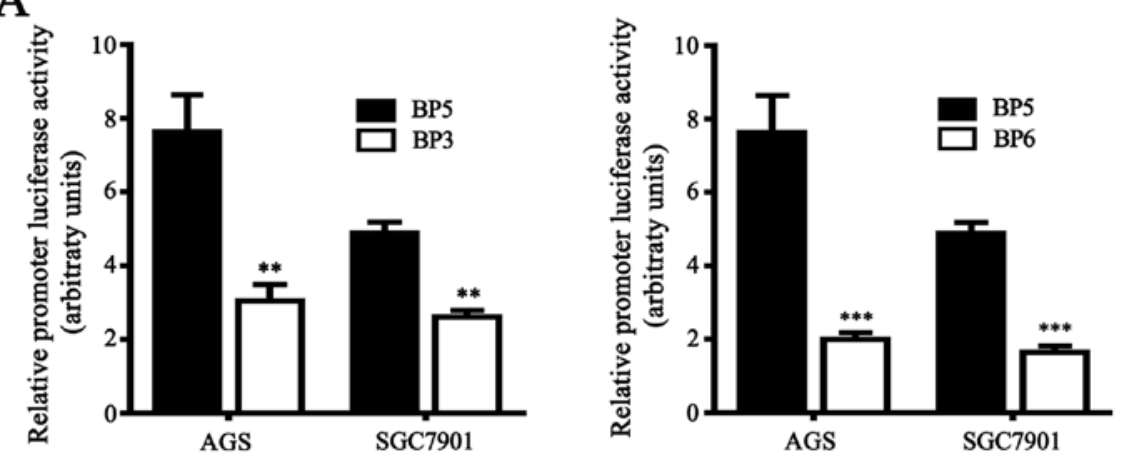

$-39$

BP5(-59/+63): GCGCGAGCCACCTCCAGGGGCGGGGCCCAGGCCGC....... CTGTCCCAGACTTTGC BP3(-34/+63): $\quad$ CCCAGGCCGC....... CTGTCCCAGACTTTGG

BP6(mutant): GCGCGAGCCACCTCCAAGTTAGAAGCCCAGGCCGC....... CTGTCCCAGACTTTGG

B
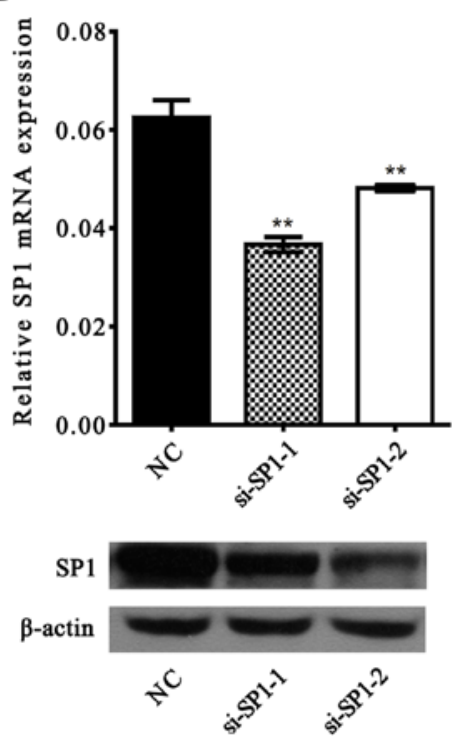

D

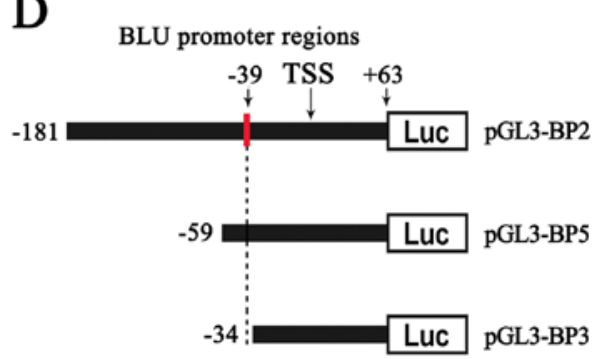

C
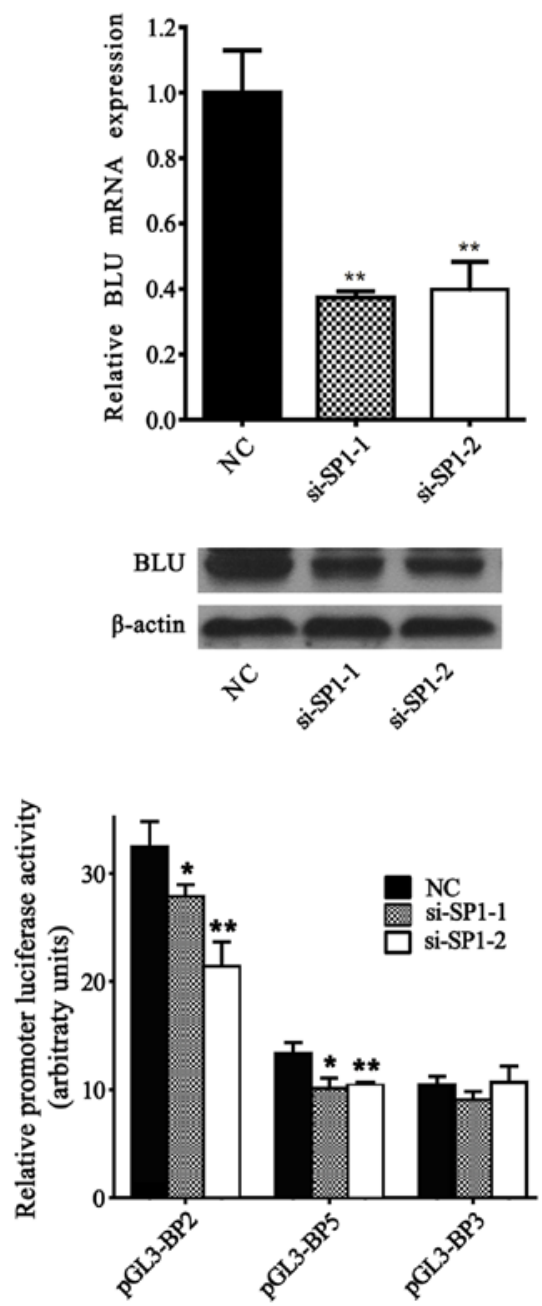

Figure 5. Sp1 activates the transcription of BLU promoter. (A) Luciferase activities of the constructs BP5 (-59/+63) containing wild-type Sp1-binding site of BLU promoter, the constructs BP3 (-34/+63) with deletion of Spl-binding site and the mutant constructs BP6 (-59/+63) in AGS and SGC7901 cells. Relative luciferase activities were determined as described in Materials and methods. (B and C) Expression of Sp1 and BLU in SGC7901 cells transfected with Sp1 siRNAs. (D) Luciferase activities of the constructs containing different lengths of BLU promoter (pGL3-BP2,-BP3 and -BP5) in SGC7901 cells co-transfected with Sp1 siRNAs (si-Sp1-1 and si-Sp1-2) or NC. The relative luciferase activities of these constructs are shown as a bar in the histograms (right panel) and each construct is shown (left panel). ${ }^{*} \mathrm{P}<0.05 ;{ }^{* *} \mathrm{P}<0.01 ;{ }^{* * * *} \mathrm{P}<0.001$.

the past decades, despite many improvements in the early detection and treatment of gastric cancer, $>50 \%$ of gastric cancers were diagnosed at advanced stages and the overall prognosis is unsatisfactory (31-34). Higher incidence rate of 

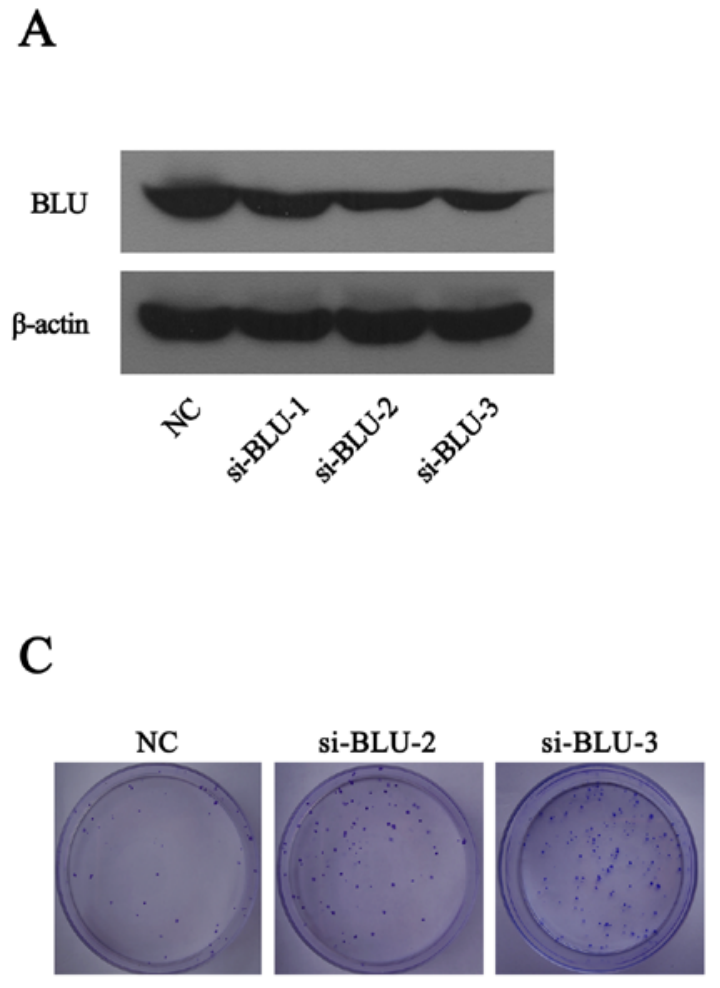

B
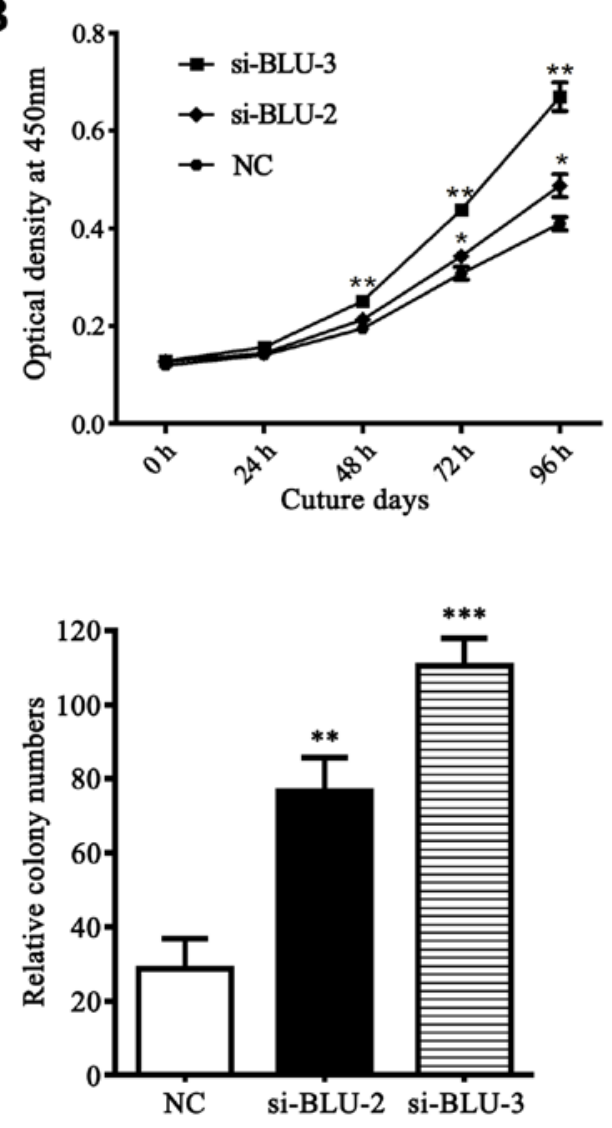

Figure 6. Knockdown of BLU promotes cellular growth and colony formation in gastric cancer cells. (A) Expression of BLU protein in SGC7901 cells transfected with BLU siRNAs (si-BLU-1, si-BLU-2 and si-BLU-3) or negative control (NC). Scrambled sequence was used as NC. (B) Cell viabilities of SGC7901 cells transfected with BLU siRNAs or NC. (C) Clonogenicity of SGC7901 cells transfected with BLU siRNAs or NC. ${ }^{*} \mathrm{P}<0.05 ;{ }^{* *} \mathrm{P}<0.01 ;{ }^{* * * *} \mathrm{P}<0.001$.

gastric carcinoma leads to even more actual problems in China (35). Therefore, to further elucidate the underlying mechanisms of gastric cancer has become an urgent requirement.

In the present study, we demonstrated that the BLU expression was commonly downregulated in gastric cancer. Moreover, silencing BLU with siRNA significantly promoted the cellular proliferation and clonogenicity, indicating that BLU served as a tumor suppressor in gastric cancer. Our results were supported by a panel of previous studies which detected the frequent loss of BLU in nasopharyngeal carcinoma, ovarian carcinoma, non-small cell lung carcinoma, glioma and esophageal cancer (18-21,36). Promoter hypermethylation has been recognized as common mechanism leading transcriptional inactivation of TSGs in many types of cancer $(37,38)$. In the present study, we found that the expression of BLU in gastric cancer was notably repressed by promoter $\mathrm{CpG}$ hypermethylation, especially by hypermethylated $-39 \mathrm{CpG}$ site which is located within Sp1-binding transcription element. Moreover, the demethylating agent treatment significantly restored the expression of BLU in gastric cancer cells. These results provided evidence that loss of BLU in gastric cancer is caused at least partly by the promoter hypermethylation. Similar phenomenon was also reported in glioma, cervical neoplasia and other malignancies $(12,17,20)$.

Subsequently, we tried to clarify the mechanisms underlying epigenetically silenced BLU expression in gastric cancer. First, we identified a functional BLU promoter which lacks a TATA-box and contains two GC boxes. As a transcription activator, Sp1 binds to GC box and regulates the basal transcription of TATA-less promoters $(26,27,39)$. Using ChIP, EMSA and luciferase reporter assays, we identified that Sp1 could specifically bind to the BLU promoter (-43/-35) and regulate its transcriptional activity. Moreover, $-39 \mathrm{CpG}$ was located within the predicted Sp1-binding sites and frequently hypermethylated in gastric cancer. In fact, DNA methylation was considered to repress epigenetically gene expression via blocking the binding of transcription factor such as E2F and Sp1 to hypermethylated $\mathrm{CpG}$ dinucleotides within TF-binding sites (22-25). Consistently, our data showed that $-39 \mathrm{CpG}$ methylation significantly reduced transcription activity of BLU by $\sim 70 \%$. Combined with ChIP and EMSA assay, the results indicate that hypermethylation of -39 CpG may decrease BLU expression via interfering with the binding of Sp1.

As previously reported, the hypermethylation progress lacks alteration in the gene sequences and can be reversed. The demethylated agent 5-Aza was able to restore the function of the TSGs silenced by promoter hypermethylation $(29,40)$. In the present study, we also observed that BLU expression was restored after treatment with 5-Aza in AGS and SGC7901 cells. In addition, we found that knockdown of BLU promoted gastric cell proliferation and clonogenicity. These results suggest that BLU may become a potential target for epigenetic therapy of gastric cancer. 
In conclusion, we identified a novel functional BLU promoter and proved that BLU promoter activity was regulated by Sp1. Furthermore, we found that hypermethylated $-39 \mathrm{CpG}$ in BLU proximal promoter directly reduced its binding with Sp1, which may be one of the mechanisms accounting for the inactivation of BLU in gastric cancer.

\section{Acknowledgements}

The authors would like to thank the patients with gastric cancer for their participation and cooperation. This study was supported in part by the grants from Natural Science Foundation of Jiangsu Province Colleges (14KJB320014), Suzhou Key Laboratory for Molecular Cancer Genetics (SZS201209) and A Project funded by the Priority Academic Program Development of Jiangsu Higher Education Institutions (PAPD).

\section{References}

1. Ferlay J, Soerjomataram I, Dikshit R, Eser S, Mathers C, Rebelo M, Parkin DM, Forman D and Bray F: Cancer incidence and mortality worldwide: Sources, methods and major patterns in GLOBOCAN 2012. Int J Cancer 136: E359-E386, 2015.

2. Jemal A, Bray F, Center MM, Ferlay J, Ward E and Forman D: Global cancer statistics. CA Cancer J Clin 61: 69-90, 2011.

3. Uemura N, Okamoto S, Yamamoto S, Matsumura N Yamaguchi S, Yamakido M, Taniyama K, Sasaki N and Schlemper RJ: Helicobacter pylori infection and the development of gastric cancer. N Engl J Med 345: 784-789, 2001.

4. Nobili S, Bruno L, Landini I, Napoli C, Bechi P, Tonelli F, Rubio CA, Mini E and Nesi G: Genomic and genetic alterations influence the progression of gastric cancer. World J Gastroenterol 17: 290-299, 2011.

5. Qu Y, Dang S and Hou P: Gene methylation in gastric cancer. Clin Chim Acta 424: 53-65, 2013.

6. Oue N, Mitani Y, Motoshita J, Matsumura S, Yoshida K, Kuniyasu H, Nakayama $\mathrm{H}$ and Yasui W: Accumulation of DNA methylation is associated with tumor stage in gastric cancer. Cancer 106: 1250-1259, 2006.

7. Jones PA and Baylin SB: The fundamental role of epigenetic events in cancer. Nat Rev Genet 3: 415-428, 2002.

8. Baylin SB and Herman JG: DNA hypermethylation in tumorigenesis: Epigenetics joins genetics. Trends Genet 16: 168-174 2000.

9. Lerman MI and Minna JD: The 630-kb lung cancer homozygous deletion region on human chromosome 3p21.3: Identification and evaluation of the resident candidate tumor suppressor genes. The International Lung Cancer Chromosome 3p21.3 Tumor Suppressor Gene Consortium. Cancer Res 60: 6116-6133, 2000.

10. Masselink $\mathrm{H}$ and Bernards R: The adenovirus E1A binding protein BS69 is a corepressor of transcription through recruitment of N-CoR. Oncogene 19: 1538-1546, 2000.

11. Wolford JK and Prochazka M: Structure and expression of the human MTG8/ETO gene. Gene 212: 103-109, 1998.

12. Agathanggelou A, Dallol A, Zöchbauer-Müller S, Morrissey C, Honorio S, Hesson L, Martinsson T, Fong KM, Kuo MJ, Yuen PW, et al: Epigenetic inactivation of the candidate 3p21.3 suppressor gene BLU in human cancers. Oncogene 22: 1580-1588, 2003.

13. Cheng Y, Ho RL, Chan KC, Kan R, Tung E, Lung HL, Yau WL, Cheung AK, Ko JM, Zhang ZF, et al: Anti-angiogenic pathway associations of the 3p21.3 mapped BLU gene in nasopharyngeal carcinoma. Oncogene: Oct27, 2014 (Epub ahead of print). doi: 10.1038/onc.2014.353 2014.

14. Zhang X, Liu H, Li B, Huang P, Shao J and He Z: Tumor suppressor BLU inhibits proliferation of nasopharyngeal carcinoma cells by regulation of cell cycle, c-Jun $\mathrm{N}$-terminal kinase and the cyclin D1 promoter. BMC Cancer 12: 267, 2012.

15. Park ST, Byun HJ, Kim BR, Dong SM, Park SH, Jang PR and Rho SB: Tumor suppressor BLU promotes paclitaxel antitumor activity by inducing apoptosis through the downregulation of Bcl-2 expression in tumorigenesis. Biochem Biophys Res Commun 435: 153-159, 2013.
16. Yoo HJ, Kim BR, Byun HJ, Park SY and Rho SB: BLU enhances the effects of anti-angiogenic activity in combination with gemcitabine-based chemotherapeutic agents. Int J Biochem Cell Biol 45: 1236-1245, 2013.

17. Lai HC, Lin YW, Chang CC, Wang HC, Chu TW, Yu MH and Chu TY: Hypermethylation of two consecutive tumor suppressor genes, BLU and RASSF1A, located at 3p21.3 in cervical neoplasias. Gynecol Oncol 104: 629-635, 2007.

18. Ito M, Ito $G$, Kondo M, Uchiyama M, Fukui T, Mori S, Yoshioka H, Ueda Y, Shimokata K and Sekido Y: Frequent inactivation of RASSF1A, BLU, and SEMA3B on 3p21.3 by promoter hypermethylation and allele loss in non-small cell lung cancer. Cancer Lett 225: 131-139, 2005.

19. Yi Lo PH, Chung Leung AC, Xiong W, Law S, Duh FM, Lerman MI, Stanbridge EJ and Lung ML: Expression of candidate chromosome 3p21.3 tumor suppressor genes and downregulation of BLU in some esophageal squamous cell carcinomas. Cancer Lett 234: 184-192, 2006.

20. Hesson L, Bièche I, Krex D, Criniere E, Hoang-Xuan K, Maher ER and Latif F: Frequent epigenetic inactivation of RASSF1A and BLU genes located within the critical 3p21.3 region in gliomas. Oncogene 23: 2408-2419, 2004.

21. Qiu GH, Tan LK, Loh KS,Lim CY, Srivastava G, Tsai ST, Tsao SW and Tao Q: The candidate tumor suppressor gene BLU, located at the commonly deleted region 3 p21.3, is an E2F-regulated, stress-responsive gene and inactivated by both epigenetic and genetic mechanisms in nasopharyngeal carcinoma. Oncogene 23: 4793-4806, 2004.

22. Zhang X, Yang R, Jia Y, Cai D, Zhou B, Qu X, Han H, Xu L, Wang L, Yao Y, et al: Hypermethylation of Sp1 binding site suppresses hypothalamic POMC in neonates and may contribute to metabolic disorders in adults: Impact of maternal dietary CLAs. Diabetes 63: 1475-1487, 2014.

23. Guo D, Wu B, Yan J, Li X, Sun H and Zhou D: A possible gene silencing mechanism: Hypermethylation of the Keap1 promoter abrogates binding of the transcription factor Sp1 in lung cancer cells. Biochem Biophys Res Commun 428: 80-85, 2012.

24. Ehrlich S, Weiss D, Burghardt R, Infante-Duarte C, Brockhaus S, Muschler MA, Bleich S, Lehmkuhl U and Frieling H: Promoter specific DNA methylation and gene expression of POMC in acutely underweight and recovered patients with anorexia nervosa. J Psychiatr Res 44: 827-833, 2010.

25. Campanero MR, Armstrong MI and Flemington EK: CpG methylation as a mechanism for the regulation of E2F activity. Proc Natl Acad Sci USA 97: 6481-6486, 2000.

26. Lania L, Majello B and De Luca P: Transcriptional regulation by the Sp family proteins. Int J Biochem Cell Biol 29: 1313-1323, 1997.

27. Emili A, Greenblatt J and Ingles CJ: Species-specific interaction of the glutamine-rich activation domains of Sp1 with the TATA box-binding protein. Mol Cell Biol 14: 1582-1593, 1994.

28. Yuan H, Gong A and Young CY: Involvement of transcription factor $\mathrm{Sp} 1$ in quercetin-mediated inhibitory effect on the androgen receptor in human prostate cancer cells. Carcinogenesis 26: 793-801, 2005.

29. Qian Q, Shi X, Lei Z, Zhan L, Liu RY, Zhao J, Yang B, Liu Z and Zhang HT: Methylated $+58 \mathrm{CpG}$ site decreases DCN mRNA expression and enhances TGF- $\beta /$ Smad signaling in NSCLC cells with high metastatic potential. Int J Oncol 44: 874-882, 2014.

30. Dynan WS and Tjian R: The promoter-specific transcription factor Sp1 binds to upstream sequences in the SV40 early promoter. Cell 35: 79-87, 1983.

31. Hundahl SA, Phillips JL and Menck HR: The National Cancer Data Base Report on poor survival of U.S. gastric carcinoma patients treated with gastrectomy: Fifth Edition American Joint Committee on Cancer staging, proximal disease, and the 'different disease' hypothesis. Cancer 88: 921-932, 2000.

32. Dikken JL, van de Velde CJ, Coit DG, Shah MA, Verheij M and Cats A: Treatment of resectable gastric cancer. Therap Adv Gastroenterol 5: 49-69, 2012.

33. Mickevicius A, Ignatavicius P, Markelis R, Parseliunas A Butkute D, Kiudelis M, Endzinas Z, Maleckas A and Dambrauskas Z: Trends and results in treatment of gastric cancer over last two decades at single East European centre: A cohort study. BMC Surg 14: 98, 2014.

34. Forman D and Burley VJ: Gastric cancer: Global pattern of the disease and an overview of environmental risk factors. Best Pract Res Clin Gastroenterol 20: 633-649, 2006.

35. Rahman R, Asombang AW and Ibdah JA: Characteristics of gastric cancer in Asia. World J Gastroenterol 20: 4483-4490, 2014. 
36. Chiang YC, Chang MC, Chen PJ, Wu MM, Hsieh CY, Cheng WF and Chen CA: Epigenetic silencing of BLU through interfering apoptosis results in chemoresistance and poor prognosis of ovarian serous carcinoma patients. Endocr Relat Cancer 20: 213-227, 2013

37. Wanajo A, Sasaki A, Nagasaki H, Shimada S, Otsubo T, Owaki S, Shimizu Y, Eishi Y, Kojima K, Nakajima Y, et al: Methylation of the calcium channel-related gene, CACNA2D3, is frequent and a poor prognostic factor in gastric cancer. Gastroenterology 135: $580-590,2008$
38. Jones PA and Laird PW: Cancer epigenetics comes of age. Nat Genet 21: 163-167, 1999.

39. Batistuzzo de Medeiros SR, Krey G, Hihi AK and Wahli W: Functional interactions between the estrogen receptor and the transcription activator $\mathrm{Sp} 1$ regulate the estrogen-dependent transcriptional activity of the vitellogenin A1 io promoter. J Biol Chem 272: 18250-18260, 1997.

40. Christman JK: 5-Azacytidine and 5-aza-2'-deoxycytidine as inhibitors of DNA methylation: Mechanistic studies and their implications for cancer therapy. Oncogene 21: 5483-5495, 2002 